\title{
Revisión de las Habilidades Socio-cognitivas en la infancia temprana
}

\section{Revision of Social-cognitive Skills in early childhood}

\author{
Chamarrita Farkas K. \\ Pontificia Universidad Católica de Chile \\ Sandra Grothusen B. \\ Instituto Alemán, Puerto Montt \\ Marie Josee Muñoz B. \\ Universidad Santo Tomás de Chile \\ Patricia von Freeden S. \\ Asociación Chilena de Seguridad, Puerto Montt \\ (Recepción: Agosto 2006 - Aceptación: Octubre 2006)
}

\begin{abstract}
La infancia temprana le genera al niño una serie de necesidades nuevas; su mundo social se amplia, aumentan sus necesidades y las personas y contextos con los que interactúa, así como las exigencias que le plantea el ambiente social. A la vez, la interacción con los demás es fuente de crecimiento y desarrollo, siendo por tanto las habilidades sociales y el ajuste social, temas de gran relevancia a esta edad.

En esta área son de suma importancia las habilidades socio-cognitivas, las cuales mediatizan la adaptación social del niño a su entorno. En este artículo se revisan aquellas habilidades que han recibido mayor apoyo teórico y empírico en esta etapa del desarrollo -resolución de problemas interpersonales, atribución de causalidad, atribución de intencionalidad y toma de perspectiva- así como cl nivel de logro esperado cn cada una de cllas.
\end{abstract}

Palabras clave: Habilidades socio-cognitivas, infancia temprana, desarrollo social.

\begin{abstract}
Early childhood generates to the child a series of new needs. His social world is expanded, his needs and the people and contexts with he interacts increase, as well as the exigencies that the social environment presents. At the same time, the interaction with others is a source of growth and development, being the social skills and social adjustment, matters of great relevance at this age.

In this area the social-cognitive skills are extremely important, by them are that the child adapts to his environment. In this article are reviewed those skills that have received more teorical and empiric backup in this stage of development -resolving interpersonal problems, cause attribution, intention attribution and choosing a perspective- and the level of achievement expected from each of them.
\end{abstract}

Keywords: Social-cognitive abilities, Early childhood, Social development.

El ser humano es por naturaleza un ser social. Existe en él una tendencia natural a establecer relaciones con otros seres humanos a lo largo de su vida, ya que el establecimiento de estas relaciones es una condición necesaria para una adecuada satisfacción de sus necesidades, desde las más básicas hasta aquellas de índole superior.

Desde que el niño nace, queda en evidencia su naturaleza social, puesto que establece una estrecha relación con otro ser humano, su madre, de quien depende absolutamente para lo-

* Correspondencia a: Chamarrita Farkas K. Av. Vicuña Mackenna 4860, Escucla de Psicología, Pontificia Universidad Católica de Chile. Santiago - Chile. Teléfonos: (56-2) 6864605, Fax (56-2) 5533092. E-mail: chfarkas@puc.cl grar la satisfacción de todas sus necesidades, por ejemplo alimentación, abrigo, cuidado, etc. A medida que el niño crece va aumentando el número de necesidades, exigencias y también el número de personas con las que va interactuando; de esta manera su desarrollo se da en estrecha relación con el ambiente social.

La interacción con los demás es fuente de crecimiento y desarrollo en la medida que le brinda al niño estimulación e información acerca de sí mismo y de la realidad que lo rodea. Esta información va siendo procesada e incorporada, estructurándose un marco de referencia que lo orientará en sus conductas futuras.

Por todo lo antes mencionado, se puede concluir que es fundamental que la persona posea las habilidades necesarias para poder procurar- 
se relaciones sociales satisfactorias, lo cual hace necesario revisar el tema del desarrollo social y la influencia que en él tienen los logros alcanzados en otros planos del desarrollo psicológico.

\section{Desarrollo Social}

El ser humano desde su nacimiento, y aun antes, desde su concepción y hasta su muerte, está sometido a un continuo proceso de desarrollo en sus distintas áreas de funcionamiento, avanzando desde estados de indiferenciación hacia una mayor complejidad y diversificación de funciones (Berwart \& Zegers, 1980). Los planteamientos de la psicología evolutiva ofrecen una visión acerca de cómo se da este desarrollo en las distintas áreas que configuran a la persona, las cuales son interdependientes e interactúan entre sí, por lo tanto los cambios observados en cualquiera de ellas van a repercutir en las demás, puesto que el ser humano es un todo integrado y funciona como tal.

En lo que se refiere al desarrollo social, ya en los primeros momentos de la vida se constata la existencia de respuestas sociales, como por ejemplo, la sonrisa social en la quinta o sexta semana de vida (Berwart \& Zegers, op. cit.).

Dentro de este continuo de desarrollo, después del primer año de edad el niño logra dos grandes adquisiciones sustentadas en la maduración biológica, que son la marcha y el lenguaje, las cuales incrementan sus posibilidades de contacto con otras personas (Berwart \& Zegers, op. cit.).

Hasta los cuatro o cinco años de edad el niño está básicamente unido a su familia, es muy dependiente de sus padres y es la familia el medio de socialización más importante. Luego el ingreso al colegio constituye para muchos niños la primera separación de la madre durante gran parte del día y casi diariamente, por lo que el colegio pasa a jugar un rol importante en ayudar al niño a disminuir sus lazos de dependencia del hogar y le ofrece al mismo tiempo dos nuevos agentes de socialización: los profesores y los compañeros de curso. A esta edad se amplía el ambiente social y se le presentan nuevas exigencias al niño (Mussen, 1982).

El ingreso y participación del niño en este ambiente social más complejo se ve facilitado por el hecho de que entre los tres años y medio y los cinco años y medio aproximadamente, se manifiesta un aumento de la sociabilidad, de la tendencia a reunirse, comunicarse, y compartir con los demás (Remplein, 1980). Al inicio de la edad escolar se constata la emergencia y/o particular acentuación de los motivos sociales, se observa una búsqueda de amigos y compañeros de juego, una necesidad de estar con otros niños y de pertenecer a un grupo. Aparecen sentimientos de lealtad, de compañerismo, y de admiración hacia sus pares. Todos estos nuevos motivos le plantean al niño una serie de tareas cuyo logro requiere alcanzar un grado satisfactorio de ajuste social. Esto implica por una parte, la adquisición de conductas y actitudes similares a las del grupo, y por otra parte, comprender el funcionamiento del grupo. De esta manera las dos metas de la socialización en esta etapa son la adquisición de roles y el aprender a modular la conducta de acuerdo a las normas de la convivencia y regulación social (Berwart \& Zegers, 1980).

Otro aspecto importante que posibilita y favorece la adaptación a las exigencias planteadas por el medio social que se amplía, son los logros en el área del desarrollo moral que se han alcanzado a esta edad. En este ámbito se espera que el niño haya alcanzado la etapa de la moral convencional, lo que implica que debe ser capaz de aceptar la norma, pero dadas las características de su pensamiento concreto no puede adoptar una posición crítica frente a ella y a los valores que la sustentan. Son centrales además valores tales como la justicia, la reciprocidad y el respeto mutuo (Berwart \& Zegers, op. cit). Estas adquisiciones en el ámbito del desarrollo moral posibilitan y favorecen las interacciones con pares y con adultos distintos a los padres.

A partir de esta edad el horizonte social se va ampliando cada vez más, planteándole al individuo nuevas y más complejas exigencias, debiendo entrar en interacción con diferentes personas y establecer relaciones significativas. Los logros alcanzados en cada etapa en los distintos planos del desarrollo psicológico influyen en la expresión conductual de las habilidades sociales y en el grado de ajuste social de la persona.

\section{Habilidades Sociales y Ajuste Social}

Los planteamientos de la teoría del aprendizaje social de Bandura (1976) sobre la conducta interpersonal sostienen que las respuestas de las personas en distintas situaciones sociales han 
sido adquiridas por experiencias previas así como de la observación del comportarse de otras personas, y son mantenidas y/o modificadas por las consecuencias sociales que derivan de la conducta propia y de la de los otros.

El individuo no es visto como un recipiente pasivo que se moviliza sólo en función de la influencia de la conducta de los demás, sino que por el contrario, se plantea una interacción recíproca en la que cada persona con su conducta puede influir en la conducta social del otro, y así en la conducta que el otro tiene hacia ella. El comportamiento de los demás retroalimenta a la persona acerca de su actuar, el cual puede mantenerse o modificarse.

La relación entre las distintas situaciones sociales a las que el individuo se ve enfrentado y las respuestas que emite frente a ellas no es automática, sino que hay una participación de los procesos cognitivos (expectativas, atribuciones, intenciones entre otros) los cuales mediatizan las respuestas de la persona en los procesos de interacción social.

Cuando una persona se enfrenta a una situación social desarrolla una serie de expectativas $\mathrm{o}$ anticipaciones acerca de la persona con la que interactúa, y también anticipa las probables consecuencias que sus propias respuestas pueden elicitar en el otro. La respuesta que el sujeto elija para interactuar será aquella que le ha dado éxito en el pasado, o que ha aprendido a través de la observación de otras personas en situaciones similares (Bandura, op. cit.). La respuesta elegida producirá ciertas consecuencias sociales, y según el éxito o fracaso de los resultados, la persona volverá o no a utilizar conductas similares en situaciones futuras (Eisler \& Frederiksen, 1980).

Las personas tienen como objetivo obtener satisfacción de la gran gama de relaciones sociales que establecen. En la sociedad contemporánea un funcionamiento exitoso con los demás requiere de cierta maestría en el desempeño social, lo que implica poder desarrollar habilidades sociales relativamente complejas. La destreza con la que un individuo es capaz de crear un clima social favorable en el cual los otros respondan de acuerdo a sus expectativas y deseos, es un parámetro que indica cuán hábil socialmente es la persona (Eisler \& Frederiksen, op. cit.).

Esta destreza implica aprender un repertorio de conductas sociales que sean efectivas, entre las cuales se pueden mencionar un adecuado conocimiento de las normas que rigen la conducta social en distintas situaciones, la habilidad para seleccionar la respuesta más efectiva entre distintas alternativas, la habilidad para percibir de manera exacta la retroalimentación de los demás y poder modificar la conducta en base a esta información, etc. En la práctica esto significa que las personas necesitan aprender a desarrollar habilidades para negociar, comprometerse, ser asertivo, escuchar de manera efectiva, usar y responder al humor, entre otras (Eisler \& Frederiksen, op. cit.). La adquisición de este repertorio conductual facilita la adaptación o ajuste de la persona a su medio social.

El ajuste social se refiere a la capacidad de poner en juego un conjunto de habilidades que le permitan al individuo enfrentarse a situaciones interpersonales. Implica un grado de eficiencia general en el ámbito interpersonal que incluye interacciones exitosas con los demás y un comportamiento que se adecue a las normas que regulan estas interacciones sociales (Ladd \& Mize, 1983).

En el logro de un adecuado ajuste social influyen tanto factores ambientales como personales. Dentro de los primeros es importante destacar el contexto en el que la persona está inserta, las posibilidades que el ambiente le ofrece para ejercitar distintas conductas, la ausencia o presencia de modelos de conducta o la exposición a modelos ajustados o desajustados. En cuanto a los factores personales se consideran las características físicas, la adecuación de la conducta en función del rol, características emocionales, ritmo y nivel de desarrollo, lenguaje, repertorio conductual, y muy especialmente las habilidades sociales que posea el individuo.

Considerando las definiciones planteadas por distintos autores, las habilidades sociales pueden ser conceptualizadas como un repertorio de conductas verbales y no verbales que permiten la interacción con los demás en los distintos contextos sociales, de maneras específicas que son socialmente aceptadas y valoradas (Combs \& Slaby, 1977; Ladd \& Mize, 1983; Rinn \& Markle, 1979).

Las habilidades sociales dependen estrechamente de la situación o contexto específico en que se da la interacción social (Eisler \& Frederiksen, 1980), se manifiestan en el plano conductual, y su expresión es mediatizada por 
factores cognitivos que reciben el nombre de "habilidades socio-cognitivas". Se considera que una persona es hábil socialmente en la medida que es exitosa en la obtención de resultados deseables así como en la evitación de las consecuencias indeseables, sin ocasionar daño a los demás y sin violar sus derechos (Eisler \& Frederiksen, op. cit.).

Eisler y Frederiksen (1980) plantean que las habilidades sociales son un constructo multidimensional, implicando una dimensión conductual, una cognitiva y una situacional. Dentro de la dimensión conductual señalan las conductas verbales y no verbales, así como las conductas motoras que acompañan al lenguaje. Dentro de la dimensión cognitiva consideran el conocimiento de alternativas de respuesta que sean adecuadas, las creencias y actitudes en relación al desarrollo de cierto tipo de conductas, la percepción de las intenciones y motivaciones del otro, y las expectativas en relación a las probables consecuencias de realizar determinadas conductas. Finalmente los autores destacan la relevancia de considerar el contexto en el cual ocurre una conducta, ya que los elementos situacionales llevan a que un comportamiento sea evaluado como adecuado o inadecuado.

Piaget e Inhelder (1969) también han postulado una estrecha relación entre lo cognitivo y lo social, en el sentido que los logros en el área cognitiva son condición necesaria -aunque no suficiente-para un adecuado desempeño social.

El implementar estrategias para enseñarle a la persona a ser más hábil socialmente puede verse como una importante vía de acción que permita ayudarle a obtener mayor satisfacción en sus interacciones interpersonales, siendo a la vez un factor protector de patologías psicológicas asociadas a un bajo nivel de ajuste social. Ello adquiere especial relevancia en el trabajo con niños, puesto que es fundamental detectar los desajustes a edades tempranas en las cuales una intervención terapeútica podría tener un mejor pronóstico, dado que las consecuencias negativas del desajuste aún no estarían tan arraigadas.

Una serie de estudios han demostrado relaciones significativas entre el grado de ajuste social y el aprendizaje y rendimiento escolar, el ajuste escolar, la deserción escolar (Hartrup, 1970), conductas delictivas, desórdenes emocionales (Michelson, Sugai, Wood \& Kazdin, 1983) y autoestima (Hamacheck, 1978).
Las investigaciones muestran que hay ciertas características que se asocian a los niños socialmente ajustados. Entre ellas se puede citar que tienden a ofrecer su ayuda, se muestran interesados por los demás, presentan mayor capacidad para enfrentar y superar obstáculos, una mayor capacidad para completar actividades por sí mismos, y muestran una mayor autonomía e iniciativa (Spivack, Platt \& Shure, 1976).

En lo que se refiere a los niños desadaptados socialmente se ha descrito un continuo de desajuste, cuyos polos serían la impulsividad y la inhibición social. Los niños inhibidos socialmente son descritos como excesivamente controlados en su conducta y en la expresión de sus emociones, especialmente la agresión (Spivack y col., op. cit.). Manifiestan además una baja frecuencia de interacción con otros, no plantean sus derechos y se conforman a los deseos de los demás, tienden a mostrarse pasivos, lentos en sus reacciones y en su discurso, generalmente son ignorados por sus pares, y tienden a experimentar sentimientos depresivos y de inadecuación (Michelson y col., 1983).

Los niños impulsivos son descritos como no cooperadores, desobedientes, peleadores, con tendencia a violar los derechos de los demás y a llamar la atención, y destructivos e impopulares entre sus pares, de quienes reciben frecuentes muestras de rechazo (Michelson y col., op. cit.).

En síntesis, el niño requiere de un repertorio de habilidades sociales para lograr ajustarse a su medio social. Este repertorio es mediatizado de manera fundamental por las habilidades socio-cognitivas, las cuales se abordarán a continuación.

\section{Habilidades Socio-Cognitivas}

Como ya ha sido señalado, las habilidades socio-cognitivas son procesos cognitivos que median entre las distintas situaciones-estímulo de carácter interpersonal y la expresión de las habilidades sociales en dicho contexto. Es decir, son procesos internos que están influyendo en la calidad del ajuste social (Shure \& Spivack, 1978).

Estas destrezas socio-cognitivas se van desarrollando en la medida que el niño va creciendo e interactuando con su medio ambiente. El grado en que se posean estas destrezas va a depender de variables individuales, ambientales, y de la interacción entre ambas. 
En los últimos años se han abordado los problemas en el ajuste social enfocando los déficits en las habilidades socio-cognitivas, lo que se ha visto como muy promisorio ya que se ataca la base del problema y los logros que se alcancen con intervenciones de este tipo son generalizables a diferentes áreas de la conducta social (Shure \& Spivack, op. cit.).

La literatura describe una serie de habilidades socio-cognitivas. Aquellas que han sido más investigadas aportando evidencia empírica de su relación con el ajuste social, y respecto de las cuales se encuentra un mayor acuerdo conceptual, son la habilidad de resolución de problemas interpersonales, las características del estilo atribucional, tanto en lo que se refiere a la causalidad de los eventos (locus de control) como a la atribución acerca de la intencionalidad de la conducta de los demás, y la habilidad para tomar la perspectiva del otro (Borke, 1972; Chandler \& Greenspan, 1972; Dodge \& Frame, 1982; Enright \& Lapsley, 1980; Galejs, 1978; Kaiser, 1975; Kaplan, Konecni \& Novaco, 1984; Milgram \& Milgram, 1975; Shure \& Spivack, 1972; Spivack y col., 1976).

\section{Habilidad de Resolución de Problemas Interpersonales}

Esta habilidad se refiere a la capacidad de generar conductas que apunten a resolver los problemas que se plantean en una situación interpersonal, lo cual implica evaluar distintas alternativas de acción y seleccionar la más adecuada, para llevarla a cabo (Pellegrini, 1985; Spivack y col., 1976).

Los autores que más han investigado esta habilidad son Spivack, Platt y Shure, quienes proponen que esta habilidad está compuesta por seis subhabilidades (1976):

- Habilidad que la persona posee para reconocer los recursos con que cuenta para relacionarse con los demás.

- Pensamiento alternativo, que es definido como "la habilidad para generar mentalmente diferentes soluciones que pueden ser utilizadas para resolver un mismo problema" (p. 19). A mayor cantidad de alternativas que la persona genere, mayores son las posibilidades que tiene de alcanzar su objetivo. Además es importante que las distintas alternativas sean relevantes para la obtención de lo que se desea, y que consideren la utilización de conductas adecuadas (e.g. no recurrir al uso de la fuerza física).

- Pensamiento medios-fines, el cual se refiere a la habilidad para planear y coordinar una serie de pasos determinados para el logro de un objetivo social, reconociendo posibles obstáculos, así como la relevancia (significación) de las distintas alternativas para el logro del objetivo.

- Pensamiento en relación a consecuencias. Es la habilidad de anticipar posibles consecuencias de un acto interpersonal, lo cual es de gran importancia en la elección de la alternativa que será implementada. Esta anticipación de consecuencias debe hacerse tanto para la propia persona como para los demás.

- Pensamiento causal. Es la habilidad de relacionar un evento con otro en el tiempo, con el fin de explicar las causas de una conducta en función de los eventos pasados en el contexto social que podrían haberla generado.

- Sensitividad a los problemas interpersonales. Esta habilidad alude a reconocer las situaciones interpersonales conflictivas, como tales. Es decir, que frente a una determinada situación la persona sea capaz de darse cuenta que el problema se presenta debido a que hay dos personas interactuando, y que constituye un problema debido a la interacción, sin ser necesariamente un problema en sí.

Una serie de estudios han demostrado que la habilidad de resolución de problemas interpersonales se correlaciona significativamente con el funcionamiento cognitivo global de la persona (Dudek, Lester, Goldberg \& Dyer, 1969; Shantz, 1975), con su competencia social, conductual y académica (Weinstein, 1969), con el entendimiento interpersonal, el estatus entre los pares, las conductas prosociales (Pellegrini, 1985) y con la toma de perspectiva social (Feffer \& Gourevich, 1960).

Otras investigaciones han demostrado una correlación de esta habilidad con el nivel socioeconómico y cultural de la persona (Shantz, 1983), no encontrándose en cambio evidencia que la variable "sexo" tenga una influencia significátiva. Además se relaciona directamente con el ajuste social, la tolerancia a la frustración y a la ambigüedad, e inversamente con los niveles de ansiedad y la tendencia a mentir (Higgins \& Thies, 1981; Muuss, 1960; Ojemann, 1967; Platt, Spivack, Altman, Altman \& Peizer, 1974; Shure \& Spivack, 1972). 
Spivack, Platt y Shure (1976) postulan que el desarrollo de esta habilidad depende tanto de factores internos, tales como un cierto desarrollo cognitivo y un estilo más reflexivo, como de factores externos al niño, entre los cuales tienen un rol importante la presencia de modelos que resuelvan adecuadamente ciertas situaciones interpersonales, $y$ la oportunidad que tenga el niño de practicar estas habilidades.

Además estos autores plantean que esta habilidad se desarrolla a través de una secuencia evolutiva. Así, los preescolares resuelven sus problemas en forma más bien rudimentaria y egocéntrica, sin una sensitividad a los problemas interpersonales ni reconociendo una causalidad social. La apreciación de las consecuencias que pueda tener una determinada conducta en las relaciones interpersonales parece no correlacionarse con el ajuste social antes de los cinco años de edad.

$\mathrm{Al}$ inicio de la escolaridad, entre los cinco y seis años, cobran más importancia en relación al ajuste social el pensamiento alternativo, el pensamiento en relación a consecuencias, el pensamiento causal y la sensitividad a los problemas interpersonales. Posteriores estudios empíricos corroboran la relevancia de las dos primeras subhabilidades con el ajuste social a esta edad; no así en el caso de las dos últimas (Shure \& Spivack, 1970, 1974, 1975; Shure, Spivack \& Jaeger, 1971; Shure, Spivack \& Powell, 1972; Shure, Newman \& Silver, 1973; Spivack \& Shure, 1975). Se observó que los niños más adaptados generaban un mayor número de alternativas de solución y que éstas eran de mejor calidad, es decir, eran relevantes a la situación y no implicaban el uso de agresión. Además, mostraban una mejor anticipación de las consecuencias de sus acciones en el plano interpersonal.

Durante la niñez media y la adolescencia pasa a tener un rol central el pensamiento medios-fines, incrementándose a la vez en forma gradual la sensitividad a las motivaciones sociales y la anticipación de consecuencias.

\section{Locus de Control o Atribución de Causalidad}

Esta habilidad se refiere a la atribución que la persona hace acerca de la localización de la causa de los hechos, en el sentido de si ésta se encuentra en la persona o en su entorno.
En la atribución externa de causalidad se adscribe la causa de los hechos a cualquier suceso externo al individuo; el ambiente que lo rodea, la persona con quien interactúa, las reglas del rol que desempeña, las recompensas o castigos que pueden surgir de la acción, la naturaleza de la tarea o la suerte. En la atribución interna la causa se ubica dentro de la persona; rasgos de personalidad, motivos, estados de ánimo, actitudes o esfuerzo (Rotter, 1990; Weiner, 1985).

Se considera como una habilidad socio-cognitiva el atribuir el resultado de las propias acciones preferentemente a variables internas más que a variables externas, con la consecuente sensación de la persona de que posee un grado de control sobre su ambiente (Kaiser, 1975).

Se ha encontrado evidencia que esta habilidad se correlaciona significativamente con la autoestima y las expectativas de autoeficacia de la persona (Kelley, 1967; Milgram \& Milgram, 1975), con su rendimiento académico (Galejs, 1978), con la persistencia en una tarea, con popularidad, con conductas constructivas y con responsabilidad (Kaiser, 1975).

Se propone que esta habilidad surge en los años preescolares y se va desarrollando en un continuo, formándose a partir de las expectativas que el niño va teniendo, donde juegan un rol importante los éxitos y fracasos, en el sentido de ir estableciendo relaciones causales entre las conductas llevadas a cabo y los resultados observados. Otro factor que influye en el desarrollo de esta habilidad es la historia previa del tipo de refuerzos aplicados preferentemente por los otros significativos en relación a la conducta del niño (Ayabe, Freese, Kyoung-Ja, Araky \& Kameoka, 1983).

Hegland y Galejs (1983) proponen que esta habilidad se desarrolla en una secuencia que sigue las siguientes etapas:

- Internalidad egocéntrica: en esta etapa el niño atribuye a sí mismo la causalidad de todos los eventos ocurridos en el entorno.

- Externalidad descentrada: aquí la atribución es externa tanto para los eventos positivos como para los negativos. Esto se produce al captar el niño que, al igual que él, otras personas pueden modificar el ambiente con su conducta y, como no es capaz de integrar aún ambas perspectivas, prima lo externo por considerar a los otros como más poderosos que él. Esta etapa tiene lugar en el niño pre- 
escolar, al encontrarse éste en un nivel de pensamiento preoperacional.

- Internalidad descentrada: en esta etapa se da una atribución interna para los eventos positivos, y externa para los negativos. Esto se va logrando en el niño escolar, en la medida que éste avanza en su desarrollo cognitivo hacia la etapa del pensamiento concreto. En el curso del desarrollo el niño va adquiriendo mayor sentimiento de control sobre su propio ambiente, mostrando una tendencia a tomar crédito por sus éxitos antes de asumir culpa por sus fracasos. Esto puede ser visto como una conducta defensiva natural en la medida que se está constituyendo el concepto de sí mismo (Milgram \& Milgram, 1975).

- Internalidad descentrada madura: en esta etapa se espera una atribución interna tanto para los eventos positivos como para los negativos, que se base en una consideración objetiva de los hechos. Las investigaciones enfatizan que el ajuste social en niños entre $\operatorname{los} 5$ y los 8 años de edad se relaciona con la habilidad para hacer este tipo de atribuciones (Galejs, 1978; Milgram \& Milgram, 1975).

Se ha visto en investigaciones que los niños con un locus de control interno son más efectivos en las relaciones interpersonales que aquellos con un locus de control externo (Nowicki, 1971). También se ha encontrado que estos niños son más populares entre sus pares, son más responsables, y presentan más conductas prosociales (Kaiser, 1975), además se les describe como más independientes y estables, y sus conductas son catalogadas como más positivas y menos desviadas dentro de la sala de clases (Galejs, 1978).

\section{Atribución de Intencionalidad}

Esta habilidad se refiere a la atribución que hace una persona respecto de los motivos de la conducta de otro, en el sentido de si ésta es accidental o intencional. Esto es de suma importancia en aquellas situaciones en que la persona ha recibido un daño o molestia. Se considera adecuada una atribución no sesgada de agresión, lo cual implica una tendencia a hacer atribuciones objetivas en cuanto a la presencia o ausencia de intención hostil en la conducta del otro (Brickmann, 1978; Dodge \& Frame, 1982).
El atribuir una intención agresiva a la conducta del otro refleja la interpretación que hace el sujeto del hecho de haber sido dañado o molestado, percibiendo selectivamente las claves hostiles de la situación, lo que ha sido explicado como un déficit socio-cognitivo específico. Investigaciones han demostrado una relación entre la tendencia a atribuir sesgadamente una intención hostil al otro, y el desajuste social, en el sentido de que existe una mayor probabilidad que la persona responda de manera agresiva en las interacciones interpersonales, en las que generalmente se da un cierto grado de ambigüedad (Brickman, 1978; Dodge \& Frame, 1982).

La atribución de intencionalidad depende de una serie de factores internos y externos a la persona; sus expectativas respecto al agresor, la severidad del daño percibido, claves ambiguas del contexto, y el propio rol en la situación (observador versus víctima), entre otros (Jones \& McGillis, 1976; Kaplan, Konecni \& Novaco, 1984).

Rule y Ferguson (1981) plantean que la evaluación de intencionalidad que se realiza sigue una secuencia; primero se evalúa la situación, para determinar la responsabilidad causal del otro en el daño recibido -hubo o no planificación de la conducta-. Si no fue planificada, se la considera accidental; si fue planificada se analiza si la intención era hacer daño o no. Si la intención fue hacer daño, se distingue si éste era un medio para obtener un fin distinto a dañar (conducta intencional benévola), o si el fin u objetivo de la conducta era agredir (conducta intencional malévola). Todo esto determina cómo se responderá en esa situación.

Entre los 5 y 7 años el niño es capaz de captar la intención en la conducta del otro (Spivack, Platt \& Shure, 1976), pero dado su nivel cognitivo, sólo logra distinguir entre una conducta accidental y una intencional, guiándose por las claves concretas de la situación. Es de gran peso la contigüidad temporal $y / o$ espacial entre la conducta del otro y su efecto, y priman criterios situacionales e inestables, por sobre los consistentes, distintivos y consensuales en la realización de la atribución (Rosenbach, Crockett \& Wapner, 1973; Rotenberg, 1982; Siegler \& Liebert, 1974).

En estudios realizados con niños agresivos, se ha demostrado una tendencia de éstos a percibir de manera selectiva las claves hostiles de 
las situaciones interpersonales, especialmente cuando el niño ha sido víctima de la agresión y la situación es ambigua. Además se ha postulado una relación directa entre la atribución de una intención hostil, y la conducta de vengarse, lo cual explicaría la agresividad de estos niños (Dodge, 1980; Dodge \& Frame, 1982).

\section{Toma de Perspectiva}

Es la habilidad de percibir el mundo desde la perspectiva de los demás (Borke, 1973; Enright \& Lapsley, 1980). Esto se desarrolla de acuerdo a una secuencia, desde un estado de egocentrismo, hasta llegar a una etapa en la cual la persona es capaz de descentrarse de su propio punto de vista para considerar adecuadamente el punto de vista de los demás.

Esta habilidad socio-cognitiva ha sido dividida en dos componentes:

\subsection{Toma de Perspectiva Cognitiva:}

La toma de perspectiva cognitiva se define como la habilidad de pensar acerca de lo que otra persona está pensando (Enright \& Lapsley, 1980). Distintos autores han planteado niveles de desarrollo para esta habilidad socio-cognitiva. Enright \& Lapsley (1980) realizan una integración de los planteamientos de una serie de autores (Byrne, 1974; Chandler, 1972; De Vries, 1970; Feffer \& Gouveritch, 1960; Selman, 1971, Selman \& Byrne, 1974), y proponen la siguiente secuencia evolutiva:

- Primera etapa: aquí el niño se encuentra en una posición egocéntrica, no siendo capaz de considerar un punto de vista diferente al propio y creyendo que los demás piensan igual que él.

- Segunda etapa: en esta etapa el niño es capaz de darse cuenta que otros pueden pensar en forma distinta a él frente a una situación, pero aún no es capaz de comprender cabalmente estos puntos de vista distintos, por lo cual tiende a mantener su propia posición frente a los hechos.

- Tercera etapa: El niño es capaz de adoptar distintas perspectivas, pero todavía no es capaz de coordinarlas simultáneamente; sólo de forma secuencial (asume primero un punto de vista y luego otro).

- Cuarta etapa: En esta etapa final se logra la capacidad de considerar diferentes perspec- tivas de manera simultánea. Además de asumir dos puntos de vista -el propio y el del otro con quien se interactúa- pueden verse ambos "desde fuera" (posición de observador externo).

\subsection{Toma de Perspectiva Afectiva o Empatia:}

La empatía - o habilidad de Toma de Perspectiva Afectiva como ha sido denominada por algunos autores como Enright y Lapsley- es la habilidad de inferir y comprender las emociones y sentimientos de otra persona. La respuesta empática además implica una reacción emocional vicaria evocada por el estado emocional percibido en el otro, aunque el énfasis en cuanto a habilidad socio-cognitiva está puesto en la comprensión de esa respuesta emocional (Enright \& Lapsley, 1980).

Siguiendo la misma secuencia planteada por Enright \& Lapsley (1980) en la toma de perspectiva cognitiva, esta habilidad se desarrolla en un continuo, partiendo de una posición egocéntrica, en la cual se es incapaz de comprender otros estados emocionales distintos al propio, y es característica del pensamiento preoperacional. En esta primera etapa el niño sí es capaz de reconocer las emociones y sentimientos que se originan en la otra persona, diferenciando inicialmente entre estados de placer y displacer, y posteriormente entre alegría, tristeza, rabia y miedo (Berwart \& Zegers, 1980; Hoffman, 1976; Selman \& Byrne, 1974).

En una segunda etapa el niño es capaz de anticipar correctamente las reacciones afectivas del otro. Ello lo logra a través de mecanismos de proyección, identificación y estereotipos (Borke, 1972). Posteriormente (inicio etapa escolar) es capaz de darse cuenta que otro puede sentir de manera distinta a él frente a un mismo hecho, comprensión que es inestable, oscilando entre mantener el punto de vista propio, y considerar el del otro (Chandler \& Greenspan, 1972).

Finalmente en una cuarta etapa el niño puede asumir múltiples puntos de vista en relación a las emociones y sentimientos de otros, de manera flexible y coordinada. Esta última etapa puede ser alcanzada en la niñez media y corresponde a una posición descentrada madura, en la cual el niño puede hacer juicios sociales más exactos y puede además anticipar lo que el otro puede estar sintiendo precisamente cuando esos sentimientos son distintos a los propios frente a 
un mismo hecho (Chandler \& Greenspan, op. cit.; Piaget \& Inhelder, 1956).

Una serie de estudios correlacionan esta habilidad en forma significativa con el ajuste social, con la conducta altruista, con la generosidad (Feshbach, 1973; Kaplan, Konecni \& Novaco, 1984; Levine \& Hoffman, 1975) y con la conducta prosocial (Hudson, Forman \& BrionMeisels, 1982). Además muestra correlaciones negativas con competitividad, agresión (Barnett, Mattews \& Howard, 1979; Kalliopuska, 1981), y delincuencia (Chandler, 1973).

\section{Discusión General}

En los últimos años los desajustes sociales se han abordado enfocando los déficits en las habilidades socio-cognitivas, lo cual se ha visto como un área bastante promisoria, ya que ataca el problema en sus raíces, y los logros alcanzados en las intervenciones son generalizables a distintas áreas de la conducta social (Shure \& Spivack, 1978).

El estudio de las habilidades socio-cognitivas cobra a la vez gran relevancia en el inicio de la etapa escolar, por los desafios que esta edad le implica al niño. Su mundo social se ha ampliado, otros agentes socializadores además de la familia han cobrado importancia, y un adecuado desarrollo de destrezas sociales se hace un requisito indispensable para el logro exitoso del ajuste social y de las metas del desarrollo. Las intervenciones en esta etapa permiten prevenir problemas en el ajuste, o trabajar estos problemas de manera precoz, previniendo las secuelas que ellos generan en la adolescencia y en el desempeño social del adulto.

En este artículo se han revisado cuatro habilidades socio-cognitivas que son las que reciben mayor apoyo tanto teórico como empírico sobre su relevancia en la niñez temprana: la resolución de problemas interpersonales, la atribución de causalidad o locus de control, la atribución de intencionalidad y la toma de perspectiva.

La habilidad de resolución de problemas interpersonales es una habilidad socio-cognitiva que tiene gran significación en la infancia temprana, ya que al ampliarse el entorno social del niño, le ofrece un adecuado esquema de enfrentamiento a los problemas interpersonales, sean estos semejantes a situaciones conflictivas que el niño ha debido resolver previamente, o bien, se trate de situaciones nuevas y desconocidas.
Spivack, Platt \& Shure (1976) postulan que la combinación de pensamiento alternativo y de pensamiento en relación a las consecuencias es el mejor índice respecto al grado de ajuste social del niño en la edad de cinco y seis años. La capacidad de generar distintas alternativas de solución frente a un problema indica una mayor flexibilidad conductual, y la anticipación de consecuencias juega un rol importante en la modulación de la conducta del niño. Todo esto cobra especial importancia a esta edad en la cual se amplía el espectro social del niño, debido a que empieza a asistir al colegio y debe poder desarrollar relaciones satisfactorias con sus pares y profesores, lo cual fortalecerá sus expectativas de autoeficacia y autoestima, asegurando el progresivo desarrollo de las habilidades ejercitadas.

Otra habilidad socio-cognitiva relevante en la infancia temprana es la habilidad de atribución de causalidad, puesto que facilita el reconocimiento por parte del niño que su conducta provoca un efecto sobre su medio ambiente, lo cual influye como ya ha sido dicho, en su autoestima y expectativas de autoeficacia. Una atribución interna implica la expectativa de poder aumentar la probabilidad de ocurrencia de una recompensa, o de disminuir la probabilidad de un evento aversivo, en la medida que se varíe la propia conducta.

Esto ha sido asociado a un sentimiento de control sobre el ambiente, el que a su vez se relaciona con la salud mental de la persona, en el sentido que al sentirse no participando en la causalidad de los eventos que ocurren, y especialmente si estos tienen un efecto negativo para el sujeto, puede conducir al desarrollo de expectativas de incontrolabilidad en relación a dichos eventos, lo que se ha asociado a sentimientos de desamparo y depresión (Abramson, Seligman \& Teasdale, 1978). Dado que los estilos atribucionales comienzan a sentar sus bases a muy temprana edad, es importante que ya en la niñez llegue a establecerse la tendencia a hacer atribuciones de causalidad interna tanto para los eventos positivos como para los negativos, considerando a la vez objetivamente a la realidad.

Debe tomarse en cuenta que en la infancia temprana esta atribución interna se realiza primeramente para los eventos positivos, y luego para los negativos. En los casos en que esto se da inversamente, se han encontrado correlaciones significativas con depresión infantil. 
También se ha revisado la habilidad de atribución de intencionalidad, en cuanto habilidad socio-cognitiva. Se espera que los niños que están iniciando su escolaridad sean capaces de captar la intención en la conducta del otro, distinguiendo entre una conducta accidental y una intencional, guiándose por las claves concretas de la situación. Los estudios realizados indican una relación entre la tendencia a atribuir intenciones agresivas a otros, y el mayor despliegue de conductas agresivas en las situaciones interpersonales. A la vez, se ha observado que aquellos niños que despliegan más conductas agresivas adquieren un estatus de agresivos dentro del grupo de pares. Estos niños inician y también reciben más agresión que otros niños, puesto que generalmente son rechazados por el grupo.

El rechazo social a una temprana edad tiene una influencia negativa en el desarrollo social del niño, puesto que puede fomentar una tendencia al aislamiento, o también puede favorecer el desarrollo de conductas desviadas en la línea de la delincuencia o transgresión de las normas que rigen la conducta social (Dodge \& Frame, 1982).

Finalmente se ha revisado la habilidad de toma de perspectiva. Se debe esperar que en la infancia temprana los niños al menos sean capaces de reconocer las cuatro emociones básicas (alegría, miedo, rabia y tristeza) en otra persona, y que sean capaces de darse cuenta que otros pueden pensar y sentir de manera diferente a él ante un mismo suceso. Sin embargo, dado el nivel de desarrollo cognitivo a esta edad (pensamiento concreto), el niño no tendría la capacidad para considerar de manera coordinada y flexible diferentes perspectivas en forma simultánea.

Esta habilidad también es un importante mediador del ajuste social, fundamental para el desarrollo y establecimiento de interacciones interpersonales adecuadas. El poder abandonar la posición egocéntrica y comenzar a considerar y entender que otros pueden ver los hechos desde puntos de vista distintos al propio, es fundamental a la edad en que el niño empieza a ir al colegio, puesto que se amplía su ambiente social y debe empezar a interactuar con otras personas, quienes no lo aceptarán de manera incondicional como lo hacen sus padres, sino que se verá obligado a modular su conducta de manera tal que logre la aceptación de parte de los demás. El que el niño pueda situarse en la perspectiva del otro le va a permitir regular su con- ducta, respetando los derechos de los demás y las normas para la adecuada convivencia social.

Además esta habilidad cobra significación en la medida que favorece el establecimiento y mantención de las relaciones interpersonales estrechas, dado que le permite a la persona una adecuada captación de las necesidades del otro y favorece la calidez, la comprensión y el compromiso personal.

\section{Referencias}

Abramson, L., Seligman, M. \& Teasdale, J. (1978). Learned helplessness in humans: Critique and reformulation. Journal of Abnormal Behavior: 87, 32-48.

Ayabe, H., Freese, A., Kyoung-Ja, K., Araky, M. \& Kameoka, K. (1983). Feedback and locus of control. The Jounal of Genetic Psychology. 143, 143-144.

Bandura, A. (1976). L'Aprentissage social. Bruselas: Picrre Mardoga.

Barnett, M., Mattews, K. \& Howard, J. (1979). Relationship between competitiveness and empathy in 6 - and 7 - ycars old. Developmental Psychology. 15, 221-222.

Berwart, H. \& Zegers, B. (1980). Psicología del Escolar: Santiago: TELEDUC.

Borke, H. (1972). Chandler and Greenspan's "Ersatz Egocentrism": a rejoinder. Developmental Psychology, 7, 107-109.

Borke, H. (1973). The development of empathy in chinese and american children between three and six years of age: a cross-cultural study. Developmental Psychology. 9, 102108.

Brickman, P. (1978). It is real. En J. Harvey, N. Ickes y R. Kidd (Eds.) New directions in attribution research. New Jersey: L. Erlbaum.

Byrne, D. (1974). The development of role-taking in adolescence, Unpublished Doctoral Dissertation, Harvard University.

Combs, M. \& Slavy, D. (1977). Social skill training with children. En B. Lahey y A. Kazdin (Eds.) Advances in clinical psychology, New York: Plenum Press.

Chandler, M. (1972). Egocentrism in normal and pathological chilhood development. En W. Hartrup y J. Witt (Eds.). Determinant of behavioral development. New York: Academic Press.

Chandler, M. (1973). Egocentrism and anti-social behavior: the assesment and training of social-perspective taking skills. Developmental Psychology: 9, 326-332.

Chandler, M. \& Greenspan, J. (1972). Ersatz Egocentrism: a reply to H. Borke. Developmental Psychology. 7, 104106.

De Vries, R. (1970). The development of role-taking as a reflected by the behavior of bright, average and retarded children in a social guessing game. Child Development, 4, 759-770.

Dodge, K. (1980). Social cognition and children's aggresive behavior. Child Development. 51, 162-170.

Dodge, K. \& Frame, C. (1982). Social-cognitive biases and deficits in aggresive boys. Child Development. 53, 620-635. 
Dudek, S.; Lester, E.; Goldberg, J. \& Dyer, G. (1969). Relationship of Piaget measures to standard intelligence and motor scales. Perceptual and Motor Skills, 28, 351362 .

Eisler, R. \& Frederiksen, L. W. (1980). Perfecting Social Skills: A Guide to Interpersonal Development. New York: Plenum Press.

Enright, R. \& Lapsley, D. (1980). Social role-taking: a review of the constructs, measures and measurements properties. Review of Educational Research. 50, 647-674.

Feffer, N. \& Gourevitch, V. (1960). Cognitive aspects of roletaking in children. Journal of Personality, 28, 283-396.

Feshbach, N. (1973). Empathy: an interpersonal process, Paper presented at American Psychology Association, Montreal.

Galejs, I. (1978). Locus of control and social interaction of preschool-children. Iowa State Journal of Research. 52, 435-441.

Hamacheck, D. (1978). Encounters with the Self. New York: Holt, Rinehart \& Winston.

Hartrup, W. (1970). Peer interaction and social organization, en P. H. Mussen (ed.) Carmichael's Manual of Child Psychology. New York: Wiley.

Hegland, S. \& Galejs, I. (1983). Developmental aspects of locus of control in preschool children. The Journal of Genetic Psychology: 143, 229-239.

Higgins, J. \& Thies, A. (1981). Problem solving and social position among emotionally disturbed boys, American Journal of Orthopsychiatry, 51, 356-358.

Hoffman, M. (1976). Empathy, role-taking, guilt and development of altruistic motives. En T. Lickona (Ed.) Moral development and behavior: Theory, research and social issues, New York: Rinehart and Winston.

Hudson, 1., Forman, E. \& Brion-Meisels, S. (1982). Roletaking as a predictor of prosocial behavior in cross-age tutors. Child Development. 53, 1320-1329.

Jones, E. \& Mc Gillis, D. (1976). Correspondent inferences and the attribution cubc: a comparative reappraisal. En J. Harvey, W. Ickes y R. Kidd (Eds.) New directions in attribution research, New Jersey: L. Erlbaum.

Kaiser, D. (1975). Internal-external control and causal attributions of own and others performance. Psychological Reports. 37, 423-426.

Kalliopuska, M. (1981). Empathy in 4-5 years olds children living orphanages. Finland: University of Helsinky, Departament of Psychology, Division of Applied Psychology.

Kaplan, R., Konccni, V. \& Novaco, R. (1984). Aggresion in Children and Youth. The Hague: Martinus Nijhoff Publishers.

Kelley, H. (1967). Attribution theory in social psychology. En D. Levine (Ed.) Nebraska symposium on motivation, Lincoln University of Nebraska Press.

Ladd, G. \& Mize, J. (1983). A cognitive social learning modcl of social skill training, Psychological Review; 90 , 127-157.

Levinc, L. \& Hoffman, M. (1975). Empathy and cooperation in 4-ycars olds. Developmental Psychology. 11, 533-534.

Michelson, L.; Sugai, D.; Wood, R. \& Kazdin, A. (1983). Social Skills Assessments and Training with Children. New York: Plenum Press.
Milgram, N. \& Milgram, R. (1975). Dimensions of locus of control in children. Psychological Reports. 37, 523-528.

Mussen, P. H. (1982). Desarrollo de la Personalidad en el Niño. México: Trillas.

Muuss, R. (1960). Mental health implications of a preventive psychiatry program in the light of reserach findings, Marriage and Family Living, 22, 150-156.

Nowicki, S. (1971). A locus of control scale for children. En B. McCandles (Ed.) Developmental aspects of locus of control expectancies: New methods and prospects. Symposium presented at the mecting of the American Psychological Association, Washington D.C.

Ojemann, R. (1967). Incorporating psychological concepts in the school curriculum, Journal of School Psychology, 5, 195-204.

Pellegrini, D. (1985). Social cognition and competence in Middle chilhood. Child Development. 56, 253-264.

Piaget, J. \& Inhelder, B. (1956). The child's concept of space. Londres: Routledge and Kegan Paul.

Piaget, J. \& Inhelder, B. (1969). Psicología del niño. Madrid: Moratas.

Platt, J.; Spivack, G.; Altman, N.; Altman, D. \& Peizer, S. (1974). Adolescent problem-solving thinking, Joumal of Consulting and Clinical Psychology, 42, 787-793.

Remplein, H. (1980). Tiatado de Psicología Evolutiva. Barcelona: Labor.

Rinn, R. \& Markle, A. (1979). Modification of social skills deficits in children. En A. Bellack y M. Hersen (Eds.) Research and practice in social skills training, New York: Plenum Press.

Rosenbach, D.; Crockett, W. \& Wapner, S. (1973). Developmental level, emotional involvement, and the resolution of inconsistency in impression formstion, Developmental Psychology, 8, 120-130.

Rotenberg, K. (1982). Development of character constancy of self and other, Child Development, 53, 505-512.

Rotter, J. (1990). Internal versus external control of reinforcement. American Psychologist, 45 (4), 489-493.

Rule, B. \& Ferguson, T. (1981). Developmental issues in attribution, moral judgement and aggression, University of Alberta and The Catholic University of Nijmegen, Holland.

Selman, R. (1971). Taking another's perspective: role-taking development in carly chilhood. Child Development, 42, 1721-1734.

Sclman, R. \& Byrnc, D. (1974). A structural-developmental analysis of levels of role-taking in middle chilhood. Child Development. 45, 803-806.

Shantz, C. (1975). The development of social cognition. En E. M. Hetherington (Ed.) Review of child development reserach, Chicago: University of Chicago Press.

Shantz, C. (1983). Social cognition. En J. Flavell y E. Markman (Eds.) Handbook of child psychology: cognitive development, New York: Wilcy.

Shure, M. \& Spivack, G. (1970). Problem solving capacity, social class and adjustment among nursery school children, Paper presented at Eastern Psychological Association, New Jersey.

Shure, M.; Spivack, G. \& Jacger, M. (1971). Problem solving thinking and adjustment among disadvantaged preschool children. Child Development, 42, 1791-1803. 


\section{CH. FARKAS / S. GROTHUSEN / M. J. MUÑOZ / P. VON FREEDEN}

Shure, M. \& Spivack, G. (1972). Means-ends thinking, adjustment and social class among elementary-schoolaged children. Journal of Consulting and Clinical Psychology, 38, 248-353.

Shure, M.; Spivack, G. \& Powell, L. (1972). A problemsolving intervention program for disadvantaged preschool children, Paper presented at Eastern Psychological Association, Boston.

Shure, M.; Newman, S. \& Silver, S. (1973). Problem-solving thinking among adjustment, impulsive and inhibited head start children, Paper presented at Eastern Psychological Association, Washington.

Shure, M. \& Spivack, G. (1974) Preschool interpersonal problem-solving (PIPS) test: Manual, Philadelphia: Department of Mental Health Sciences, Hahnemann Comunity Mental Health/Mental Retardation Center.

Shure, M. \& Spivack, G. (1975). A menatl health program for preschool and kindergarten children and a mental health program for mothers of young children: an interpersonal problem-solving approach toward social adjustment, A comprehensive report of research and training, $\mathrm{N}^{\circ} \mathrm{MH}-20372$, Washington D. C.: National Institute of Mental Health.

Shure, M. \& Spivack, G. (1978). Problem Solving Techniques in Childrearing. London: Jossey-Bass Pub.

Siegler, R. \& Liebert, R. (1974). Effects of contiguity, regularity and age on children's causal inferences, Developmental Psychology, 10, 574-579.

Spivack, G. \& Shure, M. (1975). Maternal childrearing and the interpersonal cognitive problem-solving ability of four-year-olds, Paper presented at the Society for Research in Child Development, Denver.

Spivack, G., Platt, J. \& Shure, M. (1976). The ProblemSolving Approach to Adjustement. Washington: JosseyBass Publishers.

Weiner, B. (1985). An attributional theory of achievement motivation and emotion. Psychology Revien; 92 (4), 548573.

Weinstein, E. (1969). The development of interpersonal competence. En D. Goslin (Ed.) Handbook of socialization theory and research, Chicago: McNally. 